It is clear from the proof that by using Laguerre and Hermite polynomials with the corresponding weight functions, one can obtain similar results for the intervals $(0, \infty)$ and $(-\infty, \infty)$.

\title{
REFERENCES
}

1. E. Hille, G. Szego, A. Tamarkin, On some generalizations of a theorem of A. Markoff, Duke Math. J. vol. 3 (1937) p. 729.

2. Whittaker-Watson, Modern analysis, Cambridge, 1935, p. 307 (IV).

Princeton UnIVERSITY

\section{TRANSCENDENCE OF CERTAIN CONTINUED FRACTIONS}

\section{G. CUTHBERT WEBBER}

Even today very little is known about which regular continued fraction expansions represent transcendental numbers. In 1851 Liouville $^{1}$ proved the transcendence of the numbers named after him, in the expansions of which the partial quotients increase very rapidly; in 1906 Maillet $^{2}$ proved the transcendence of certain expansions in which the partial quotients may be bounded. C. L. Siegel ${ }^{3}$ proved that if the partial quotients of the expansion form an arithmetic progression of order one, the number represented is transcendental. In this paper well known transformations of continued fractions are applied, in conjunction with the Siegel theorem, to prove the transcendence of certain classes of continued fraction expansions.

We shall use the notation

$$
b_{0}+\frac{a_{1}}{b_{1}}+\frac{a_{2}}{b_{2}}+\cdots
$$

to represent a continued fraction expansion, with the partial numerators and denominators $a_{j}$ and $b_{j}$ respectively. The $j$ th convergent is denoted by $A_{j} / B_{j}$. When the expansion is regular the following notations are also used:

Received by the editors April 6, 1944.

${ }^{1} \mathrm{~J}$. Liouville, Sur des classes très-étendues de quantités dont la valeur n'est ni algêbrique, $n$ même reductible d̀ des irrationnelles algébrique, J. Math. Pures Appl. vol. 16 (1851).

2 E. Maillet, Introduction a la théorie des nombres transcendants, Paris, 1906.

3 C. L. Siegel, Über einige Anwendungen diophantischer Approximationen, Abhandlung der Preussischen Akademie der Wissenschaften, Physikalische-Mathematische Klasse, no. 1 (1929) p. 29. 


$$
\left[b_{0}, b_{1}, b_{2}, \cdots\right]=\left[b_{i}\right]_{j=0}^{\infty} \text {. }
$$

In the expansions considered $a_{j}= \pm 1(j \geqq 1)$, the partial quotients are integers, $b_{j} \geqq 1(j \geqq 1), b_{j}+a_{j+1} \geqq 2$ for an infinite set of values of $j$, and $b_{j}+a_{j+1} \geqq 1$ for all $j$ except, possibly, a finite set. Thus the expansions are semi-regular, at least after a finite number of terms, and so they converge. ${ }^{4}$ The expansions considered in the theorems are of Hurwitz type except when one or more partial numerators are -1 in Theorem I.

Siegel, in the paper referred to above, states that the methods applied therein suffice to prove that if $x \neq 0$ is algebraic, then there does not exist between $J_{\lambda}(x)$ and $J_{\lambda-1}(x)$ a rational equation with rational coefficients, if $\lambda$ is rational but not the half of an odd integer $\left(J_{\lambda}(x)\right.$ is a Bessel function of type one); also, that the same result holds when $\lambda$ is half an odd integer, due to the special form of $J_{\lambda}(x) / J_{\lambda-1}(x)$ in terms of $e^{i x}$ and to the Lindemann theorem: The form $\sum_{\nu=1}^{n} \xi_{\nu} e^{\eta_{\nu}}$ is transcendental in each non-trivial case, if $\xi_{1}, \cdots, \xi_{n}$ and $\eta_{1}, \cdots, \eta_{n}$ are algebraic numbers.

The theory of Bessel functions gives the result that

$$
\lambda x+\frac{a_{1}}{(\lambda+1) x}+\frac{a_{2}}{(\lambda+2) x}+\cdots
$$

is equal to $i J_{\lambda-1}(2 i / x) / J_{\lambda}(2 i / x)$ or $J_{\lambda-1}(2 / x) / J_{\lambda}(2 / x)$, according as the $a_{j}$ are all +1 or all -1 . If $x$ and $\lambda$ are chosen such that $x=d$ and $\lambda d=r, r$ and $d$ being positive integers, $r \geqq 2$, then

$$
r+\frac{a_{1}}{(r+d)}+\frac{a_{2}}{(r+2 d)}+\cdots
$$

where the $a_{j}$ are all +1 or all -1 , is transcendental. ${ }^{5}$

THEOREM I. The number $\xi$, represented by

(2) $\xi=g_{0}+\frac{a_{1}}{b_{1}}+\cdots+\frac{a_{k-1}}{b_{k-1}}+\frac{f_{1}}{g_{1}}+\frac{a_{1}}{b_{1}}+\cdots+\frac{a_{k-1}}{b_{k-1}}+\frac{f_{2}}{g_{2}}+\cdots$

is transcendental when the sequence $\left\{g_{j}\right\}$ is an arithmetic progression of first order whose elements are integers which are positive except, possibly, $g_{0} ; b_{1}, \cdots, b_{k}$ are positive integers, $a_{m}= \pm 1(m=1, \cdots, k-1)$,

' O. Perron, Die Lehre von den Kettenbritchen, B. G. Teubner, Leipzig, 1929, pp. 148-154.

- If the $a_{j}$ are all +1 , this becomes the Siegel theorem stated in the introduction. 
the $f_{j}$ are either all +1 or all -1 , and $g_{1}+a_{1} \geqq 1, b_{m}+a_{m+1} \geqq 1$, $b_{k-1}+f_{j} \geqq 1$, for $m=1, \cdots, k-2$ and $j \geqq 1$.

In the proof we may consider $g_{0} \geqq 2$; otherwise, the first $k+1$ or $2 k+2$ terms are dropped and the remaining terms renumbered to insure convergence of the semi-regular expansion. This does not affect the algebraic character of the whole expansion. The continued fraction (2) is transformed by "contraction" to the continued fraction with convergents $A_{j k-1} / B_{j k-1}(j=1,2,3, \cdots)$. This gives

$$
\begin{aligned}
\xi & =\frac{1}{B_{k-1}}\left\{g_{0} B_{k-1}+a_{1} B_{k-2,1}+\frac{(-1)^{k-1} a_{1} a_{2} \cdots a_{k-1} f_{1}}{\left(g_{1} B_{k-1}+f_{1} B_{k-2}+a_{1} B_{k-2,1}\right)}\right. \\
& \left.+\frac{(-1)^{k-1} a_{1} \cdots a_{k-1} f_{2}}{\left(g_{2} B_{k-1}+f_{2} B_{k-2}+a_{1} B_{k-2,1}\right)}+\cdots\right\} \\
= & \frac{1}{B_{k-1}}\left\{g_{0} B_{k-1}+a_{1} B_{k-2,1}+\frac{(-1)^{k-1} a_{1} a_{2} \cdots a_{k-1} f_{1}}{\eta}\right\}, 7
\end{aligned}
$$

where $\eta$ is a continued fraction of type (1), at least after a finite number of terms. The latter case may occur when either or both of $a_{1}$ and $f_{1}$ are -1 . Since $\eta$ is transcendental it follows that $\xi$ is also transcendental.

It is interesting to note that if the expansion is regular, the partial quotients may be made to increase as "slowly as desired" by choosing $b_{j}=1(j=1,2, \cdots, k)$ and $k$ large.

THEOREM II. If $a, d$ and $c$ are positive integers, either $c$ or $d$ being even, and $b=c^{2} a+(1 / 2) c^{2} d, e=c^{2} d$, then

$$
\alpha=[a, b, a+d, b+e, a+2 d, b+2 e, \cdots]
$$

is a transcendental number.

From the theory of continued fractions we obtain the following result: ${ }^{8}$

$$
\begin{aligned}
& c_{0} b_{0}+\frac{c_{0} c_{1} a_{1}}{c_{1} b_{1}}+\cdots+\frac{c_{k-1} c_{k} a_{k}}{c_{k} b_{k}}+\cdots \\
& \quad=c_{0}\left[b_{0}+\frac{a_{1}}{b_{1}}+\cdots+\frac{a_{k}}{b_{k}}+\cdots\right] .
\end{aligned}
$$

B O. Perron, Die Lehre von den Kettenbriichen, B. G. Teubner, Leipzig, 1929, pp. 197-203.

${ }^{7}$ By $B_{k-2,1}$ is meant the result of advancing by one the subscripts of all the $a_{j}$ and $b_{j}$ in $B_{k-2}$.

${ }^{8}$ O. Perron, Die Lehre von den Kettenbrilchen, B. G. Teubner, Leipzig, 1929, p. 196. 
Application of this result with $a_{k}=1, c_{k}=1 / c_{k-1}, c_{0}=c, c_{2 k}=c$ $(k=1,2,3, \cdots)$ to $\alpha$ yields

$$
\begin{aligned}
c \alpha= & c\left[a, c^{2} a+(1 / 2) c^{2} d, a+d, c^{2} a+(3 / 2) c^{2} d,\right. \\
& \left.a+2 d, c^{2} a+(5 / 2) c^{2} d, \cdots\right] \\
= & c c a, c a+(1 / 2) c d, c a+c d, c a+(3 / 2) c d, \\
& c a+2 c d, c a+(5 / 2) c d, \cdots] .
\end{aligned}
$$

Since the partial quotients in $c \alpha$ form an arithmetic progression of order one with common difference $(1 / 2) c d, c \alpha$, and in turn $\alpha$, is transcendental by the Siegel theorem stated in the introduction.

In the last theorem we proved the transcendence of a class of regular continued fractions in which the alternating partial quotients form an arithmetic progression of order one. The following theorem gives two more classes of a similar type.

THEOREM III. The numbers $\beta$ and $\gamma$, where

$$
\beta=\left[1+4 j,(3+4 j) u^{2}\right]_{j=0}^{\infty} \text { and } \gamma=\left[1,(3+4 j) u^{2}-2,1,3+4 j\right]_{j=0}^{\infty} \text {, }
$$

$u^{2}$ being a positive integer, are transcendental.

Again appealing to well established theory of continued fractions ${ }^{9}$ we have

$$
\delta=0+\frac{z}{1}+\frac{a_{2} z^{2}}{3}+\frac{a_{3} z^{2}}{5}+\frac{a_{4} z^{2}}{7}+\cdots=\frac{e^{z}-e^{-z}}{e^{z}+e^{-z}} \text { or } \tan z,
$$

according as the $a_{j}$ are all +1 or all -1 . Apply (3) with $c_{0}=1 / z$, $c_{2 j+1}=1, c_{2 j+2}=1 / z^{2}(j=0,1,2, \cdots)$ and $z=1 / u$; this yields

$$
\begin{aligned}
\delta & =z\left[0+\frac{1}{1}+\frac{a_{2}}{\left(3 / z^{2}\right)}+\frac{a_{3}}{5}+\frac{a_{4}}{\left(7 / z^{2}\right)}+\frac{a_{5}}{9}+\cdots\right] \\
& =(1 / u)\left[0+\frac{1}{1}+\frac{a_{2}}{3 u^{2}}+\frac{a_{3}}{5}+\frac{a_{4}}{7 u^{2}}+\frac{a_{5}}{9}+\cdots\right] \\
& =(1 / u)(1 / \beta) \text { or }(1 / u)(1 / \epsilon),
\end{aligned}
$$

according as the $a_{j}$ are all +1 or all -1 , where $\epsilon$ is defined by the above equality.

Since $u^{2}$ is a positive integer, $u$ is an algebraic number not equal to 0 , and thus $z$ is an algebraic number. Now by the Lindemann theo-

'O. Perron, p. 353. 
rem, $\tan z$ and $\left(e^{z}-e^{-z}\right) /\left(e^{z}+e^{-z}\right)$ are transcendental when $z \neq 0$ is algebraic. This proves the transcendence of $\beta$ and $\epsilon$.

Convert the semi-regular continued fraction for $\epsilon$ to a regular continued fraction $;^{10}$ this gives

$$
\begin{gathered}
\frac{1}{\epsilon}=\frac{1}{1}-\frac{1}{3 u^{2}}-\frac{1}{5}-\frac{1}{7 u^{2}}-\frac{1}{9}-\cdots, \\
\epsilon=0+\frac{1}{1}+\frac{1}{\left(3 u^{2}-2\right)}+\frac{1}{1}+\frac{1}{3}+\frac{1}{1} \\
\quad+\frac{1}{\left(7 u^{2}-2\right)}+\frac{1}{1}+\frac{1}{7}+\cdots, \\
\frac{1}{\epsilon}=\left[1,(3+4 j) u^{2}-2,1,3+4 j\right]_{j=0}^{\infty}=\gamma .
\end{gathered}
$$

Since $\epsilon$ is transcendental, so is $\gamma$.

The University of Delaware

${ }^{10}$ O. Perron, p. 159. 